\title{
Modelos de mediación sociofamiliares en el desarrollo de la competencia digital ${ }^{1}$
}

\section{Socio-Familial Mediation Models in Developing Digital Competence}

\author{
Sonia Casillas-Martín (1) https://orcid.orq/0000-0001-5304-534X \\ Marcos Cabezas-González (1) https://orcid.org/0000-0002-3743-5839 \\ Ana García-Valcárcel Muñoz-Repiso (1) https://orcid.org/0000-0003-0463-0192 \\ Verónica Basilotta-Gómez-Pablos (2) https://orcid.ora/0000-0003-1976-4548 \\ (1) Universidad de Salamanca \\ (2) Universidad a Distancia de Madrid \\ (Recibido: 4 de febrero de 2020; Aceptado para su publicación: 3 de agosto de 2020)
}

Cómo citar: Casillas-Martín, S., Cabezas-González, M., García-Valcárcel, A. y Basilotta-Gómez-Pablos, V. (2021). Modelos de mediación sociofamiliares en el desarrollo de la competencia digital. Revista Electrónica de Investigación Educativa, 23, e26, 1-18. https://doi.org/10.24320/redie.2021.23.e26.3839

\section{Resumen}

En este artículo se presenta un trabajo que pretende proponer modelos de mediación que expliquen la influencia de variables sociofamiliares en la evaluación de la competencia digital, en el área de comunicación y colaboración, de estudiantes españoles de Educación Obligatoria (12-16 años). Se empleó una metodología cuantitativa no experimental con un diseño de corte explicativo y transversal, y se trabajó con una muestra de 609 estudiantes de 18 centros educativos de acuerdo con un tipo de muestreo aleatorio estratificado. Para la recolección de información se utilizó una prueba objetiva de evaluación de conocimientos y habilidades, y una escala tipo Likert para las actitudes. Se realizaron análisis de regresión mediante la creación de modelos teóricos de referencia, basados en la técnica bootstrapping. Los resultados permiten demostrar, por medio de diferentes modelos, la influencia de variables sociofamiliares en la adquisición y desarrollo de esta competencia.

Palabras clave: habilidades digitales, tecnología educativa, tecnología de la comunicación, educación básica

\section{Abstract}

This study proposes mediation models to explain the influence of socio-familial variables in evaluating digital competence in communication and collaboration among Spanish students in compulsory education (ESO; 12-16 years of age). A non-experimental quantitative methodology was employed, within an explanatory, cross-sectional design, with a sample of 609 students from 18 schools, selected by stratified random sampling. Data was collected using an objective assessment test of knowledge and skills, and a Likert scale for attitudes. Regression analyses were performed by creating theoretical reference models with bootstrapping. The results show, through different models, the influence of socio-familial variables in acquiring and developing digital competence.

\footnotetext{
${ }^{1}$ Este artículo es resultado del proyecto I+D “Evaluación de la competencia digital de los estudiantes de educación obligatoria y estudio de la incidencia de variables sociofamiliares", financiado por el Ministerio de Economía y Competitividad del Gobierno de España dentro del Programa Estatal de Fomento de la Investigación Científica y Técnica de Excelencia, cuya finalidad es evaluar la competencia digital de estudiantes de Educación Obligatoria.
} 
Keywords: digital skills, educational technology, communication technology, basic education

\section{Introducción}

En las denominadas competencias del siglo XXI (Erstad y Voogt, 2018) una de las piezas fundamentales es la competencia digital (Siddiq et al., 2016), ya que se considera esencial para recuperar у рrocesar información, así como para el rendimiento académico y el éxito profesional (Pagani et al., 2016).

Son diferentes los términos utilizados para identificar y analizar las capacidades que los estudiantes deberían ser capaces de desarrollar con las TIC: alfabetización digital (Sefton et al., 2009), alfabetización mediática (Erstad, 2010) y competencia digital (Hatlevik et al., 2015), entre los más utilizados. Según Calvani et al. (2008), los términos competencia digital y alfabetización digital se utilizan, a menudo, como sinónimos, porque en mayor o menor medida se superponen y los conceptos que subyacen tienen muchas similitudes.

En la recomendación C 189, la Unión Europea (2018) reconoce a la competencia digital como una de las ocho competencias clave para el aprendizaje permanente, y la define como:

El uso seguro, crítico y responsable de las tecnologías digitales para el aprendizaje, en el trabajo y para la participación en la sociedad, así como la interacción con éstas. Incluye la alfabetización en información y datos, la comunicación y colaboración, la alfabetización mediática, la creación de contenidos digitales (incluida la programación), la seguridad (incluido el bienestar digital y las competencias relacionadas con la ciberseguridad), asuntos relacionados con la propiedad intelectual, la resolución de problemas y el pensamiento crítico. (p. 30).

Existen diferentes estándares, entendidos como constructos teóricos de referencia que son útiles para realizar acciones en un ámbito determinado (UNESCO, 2008), para desarrollar y evaluar esta competencia. En Europa el proyecto DigComp ${ }^{2}$ se ha convertido en un referente para el desarrollo del marco europeo de la competencia digital. Su versión más actual es DigComp 2.1, marco de competencia para los ciudadanos (Carretero et al., 2017); este modelo está estructurado en cinco áreas (información y alfabetización informacional, comunicación y colaboración, creación de contenidos digitales, seguridad, y resolución de problemas), con un total de 21 competencias digitales organizadas por niveles (básico, intermedio, avanzado y altamente especializado).

En los últimos años, algunas investigaciones sobre la evaluación de la competencia digital de los estudiantes (Colás et al., 2017; Lorenz et al., 2019; Rolf et al., 2019; Zakrajšek, 2018) confirmaron que las TIC son un instrumento fundamental en la relación persona-conocimiento (Álvarez-Quiroz y Vélez, 2014) y que es necesario apoyar y desarrollar la competencia digital en las aulas, así como revertir la brecha digital.

En cada momento histórico ha existido desigualdad en el acceso a la tecnología que ofrece la información y el conocimiento, porque siempre ha habido colectivos que por razones de género, edad, clase social o economía tienen más fácil acceso a los medios que ofrecen la información (Cabero y Ruíz-Palmero, 2018). Pero el gran problema que se enfrenta en la actualidad es que las TIC han adquirido tal significación social que no acceder a ellas se convierte en motivo de exclusión social, o más bien de e-exclusión, es decir, "que la separación y marginación meramente tecnológica se está convirtiendo en separación y marginación social y personal" (Cabero, 2015, p. 161).

Diversas investigaciones vienen a confirmarlo: un estudio de Varela (2015), realizado para determinar las principales razones de la brecha digital en España, indica la existencia de tipologías de brechas digitales: por cuestión de género, por motivo de edad, por cuestiones de formación (analfabetismo tecnológico), de índole funcional (discapacidad física o psicológica), por cuestión de renta o económicas, de índole geográfica o territorial (ausencia de infraestructura en un determinado lugar), de tipo urbana-rural y de índole formativa-laboral. Otras investigaciones, como la de Hargittai (2010), indican que los usos más beneficiosos y avanzados de Internet están directamente relacionados con variables como la edad, el nivel

\footnotetext{
2 Proyecto orientado a la identificación y validación a escala europea de los componentes clave de la competencia digital.
} 
de estudios, el género y, especialmente, con variables específicas de las TIC -como las competencias digitales y el contexto en el que se usan las tecnologías.

Por su parte, Torres (2017) utilizando datos del Instituto Nacional de Estadística (INE) obtenidos por medio de la Encuesta sobre Equipamiento y Uso de Tecnologías de la Información y Comunicación en los hogares, concluye que:

La brecha digital continúa activa en España y, lejos de desaparecer, persiste entre los grupos más y menos favorecidos... El análisis del caso español plantea un escenario de desigualdad digital específicamente referido a los distintos usos ventajosos de Internet que van apareciendo, una vez que la red ha alcanzado su actual condición estratégica y hegemónica. (pp. 31-32)

Por todo ello, es importante conocer el efecto que pueden tener diferentes variables en esta competencia para favorecer la creación de políticas de integración de las TIC en los colegios. En este sentido, se han realizado trabajos que estudian esta influencia: Li y Ranieri (2010), en el contexto chino, indicaron que factores como la edad y la escuela en la que se estudia parecen tener un impacto en la competencia digital de los estudiantes adolescentes de noveno grado, además de que vivir en un entorno digital no implica ser digitalmente competente; en el contexto noruego, Hatlevik et al. (2014) mostraron que la motivación y los antecedentes familiares, en términos de integración lingüística y número de libros en el hogar, eran predictores del grado de competencia digital de los alumnos de séptimo grado; Punter et al. (2017), utilizando los datos del International Computer and Information Literacy Study (ICILS, 2013), concluyeron que aún siguen existiendo diferencias de género en el desarrollo de la competencia digital en los adolescentes. Fernández Mellizo y Manzano (2018), en el contexto español, constataron cómo el acceso a los recursos tecnológicos fuera del colegio, consecuencia del nivel socioeconómico de los padres, está relacionado de forma positiva con la competencia digital de los estudiantes de educación secundaria y bachillerato. En el contexto coreano, Kim et al. (2018) verificaron que los estudiantes universitarios que viven en un ambiente digitalmente enriquecido en el hogar y en la escuela son más propensos a adoptar, de manera eficaz, las tecnologías digitales para el aprendizaje. En Kosovo, Shala y Grajcevci (2018) demostraron que en el desarrollo de la competencia digital de los universitarios influyen positivamente la inclusión en entornos académicos, una buena posición socioeconómica y residir en áreas urbanas frente a rurales.

\section{Método}

Se empleó una metodología cuantitativa (prueba objetiva y escala de actitudes) no experimental, con un diseño de corte explicativo y transversal, la información se recogió durante el curso académico 2018-2019.

El objetivo de la investigación fue presentar modelos de mediación que identificaran la influencia de variables sociofamiliares en la evaluación de la competencia digital de estudiantes españoles de Educación Obligatoria (12-16 años) en el área competencial de comunicación y colaboración, teniendo en cuenta sus conocimientos, habilidades y actitudes.

Las hipótesis planteadas fueron:

H1: Los estudiantes, que pertenecen a familias de nivel cultural y económico alto en las que existe un mayor número de dispositivos tecnológicos y en las que se hace un mayor uso de los mismos, estarán estimulados para una mejor adquisición de la competencia digital en el área de comunicación y colaboración (modelo de análisis de mediación).

H2: Los alumnos, que pertenecen a familias estructuradas con un nivel cultural y económico alto y en las que los hijos están integrados socialmente en el contexto educativo, tendrán una mejor competencia digital en el área de comunicación y colaboración (modelo de análisis de moderación).

H3: Los estudiantes, pertenecientes a familias con mejor nivel cultural y económico que disponen de mayor número de dispositivos y que por ello usan más la tecnología, tendrán una competencia digital en el área de comunicación y colaboración mejor, cuando existan unas condiciones adecuadas de integración social 
del estudiante y las familias sean estructuradas (modelo de análisis de mediación moderada).

La evaluación se realizó en dos provincias de la Comunidad Autónoma de Castilla y León (España), cuya población es de 7653 estudiantes (4 175 de Educación Primaria y 3478 de Educación Secundaria). Se utilizó un tipo de muestreo aleatorio estratificado (Casal y Mateu, 2003) con un margen de error de $\pm 3.8 \%$ para un nivel de confianza del 95\%, lo que llevó a trabajar con una muestra de 609 alumnos de entre 12 y 16 años, de 18 centros educativos (514 de último curso de Educación Primaria y 95 de primer curso de Educación Secundaria Obligatoria), que fueron los que finalmente realizaron de manera voluntaria la prueba, resultando una participación más alta del alumnado de Primaria que del de Secundaria. Desde el punto de vista del género se cuenta con una muestra equilibrada (51.2\% femenino y $48.8 \%$ masculino), como se muestra en la Tabla 1.

Tabla 1. Distribución de la muestra

\begin{tabular}{c|cc|cc|cc|cc}
\hline \multicolumn{4}{c}{ Curso } & \multicolumn{4}{c}{ Género } \\
\hline Muestra total & \multicolumn{4}{c|}{ 60. Primaria } & \multicolumn{1}{c}{10. Secundaria } & \multicolumn{2}{c}{ Femenino } & \multicolumn{2}{c}{ Masculino } \\
\hline $\mathrm{N}$ & $\mathrm{N}$ & $\%$ & $\mathrm{~N}$ & $\%$ & $\mathrm{~N}$ & $\%$ & $\mathrm{~N}$ & $\%$ \\
\hline 609 & 514 & 84.4 & 95 & 15.6 & 312 & 51.2 & 297 & 48.8 \\
\hline
\end{tabular}

Para su diseño, el trabajo se organizó en tres fases:

Fase 1. Revisión documental. Técnica que consiste en seleccionar y recopilar información por medio de la lectura y crítica de documentos y materiales bibliográficos (Ávila, 2006). Se llevó a cabo una revisión documental sobre las dimensiones de la competencia digital, con la intención de formular un modelo de indicadores en el cual basar el diseño de la prueba de evaluación.

Fase 2. Elaboración de un modelo de indicadores de la competencia digital. Los investigadores tomaron como referencia la estructura del modelo DigComp 2.1, para el área competencial de comunicación y colaboración se plantearon 69 indicadores organizados en tres ámbitos y tres niveles, adaptados a la población objeto de estudio (Figura 1).

Figura 1. Estructura de indicadores área 2 comunicación y colaboración

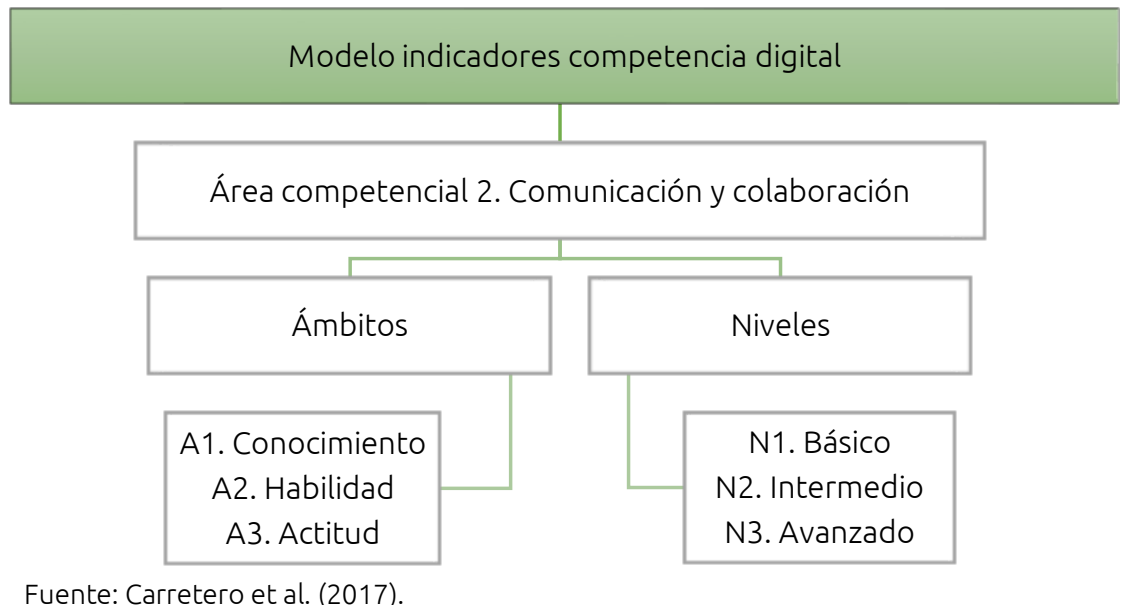

Estos indicadores pueden consultarse en García Valcárcel et al. (2019), quienes recogen un total de 325 distribuidos entre las cinco áreas de la competencia digital. 
Para la validación del contenido del modelo de esta área competencial se siguió el método de jueces. Un total de 17 expertos en el diseño de indicadores de evaluación, en competencia digital y profesionales en ejercicio de la etapa educativa de Educación Obligatoria, pertenecientes a distintos contextos educativos (Educación Obligatoria, universidad, gestión educativa), evaluaron la importancia, pertinencia y claridad de los indicadores por medio de un cuestionario en línea de escala Likert de 4 grados ( 4 = mucha, 3 = bastante, 2 = poca, 1 = ninguna).

Fase 3. Diseño y aplicación de la prueba de evaluación. Teniendo en cuenta el modelo de indicadores y los criterios para la elaboración de instrumentos de recogida de información (McMillan y Schumacher, 2005), se diseñó un banco de ítems para el área de comunicación y colaboración. Para los conocimientos y las habilidades, una prueba objetiva con preguntas que presentaban situaciones en las que los estudiantes tenían que tomar decisiones, seleccionando una respuesta correcta de entre cuatro opciones posibles. Рara las actitudes, una escala tipo Likert que va de 1 (muy en desacuerdo) a 5 (muy de acuerdo) compuesta por 6 enunciados referidos a una afirmación sobre el área competencial (Figura 2).

Figura 2. ítems de actitudes área de comunicación y colaboración

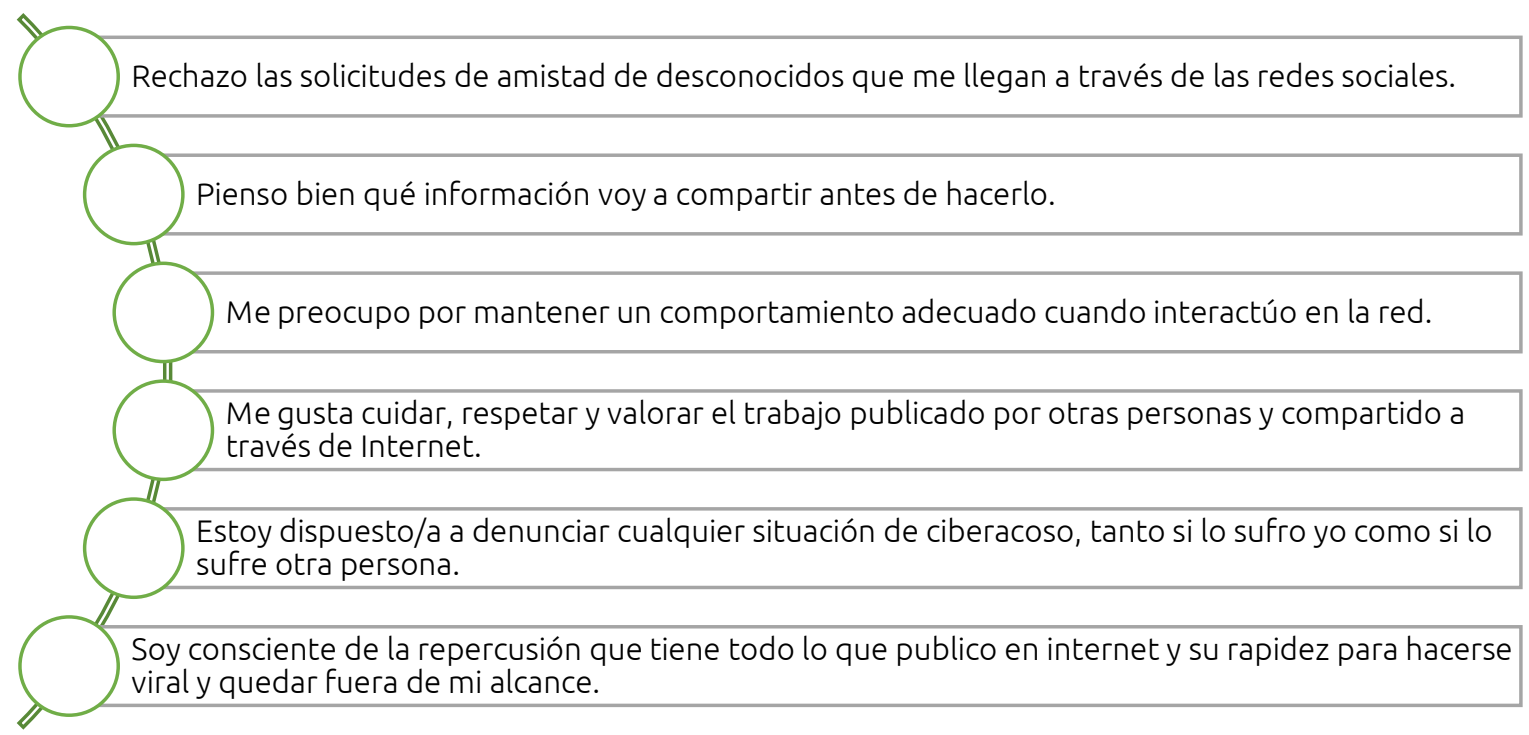

Para recoger información de tipo sociofamiliar se elaboró una escala compuesta por 17 ítems. Esta batería fue depurada por expertos, diseñándose la prueba de evaluación que fue aplicada a una muestra piloto de 288 alumnos de Educación Obligatoria. Para constatar la fiabilidad y consistencia interna se utilizó el estadístico a de Cronbach para todos los ítems (conocimiento, habilidad y actitud) arrojando éste una puntuación de 0.70 . Respecto a la validez, se optó por realizar una de contenido y de estructura utilizando la técnica de expertos, participaron como jueces los 10 miembros del equipo de investigación. Con los resultados obtenidos se diseñó la prueba definitiva (Tabla 2).

Tabla 2. Estructura de la prueba de evaluación definitiva

\begin{tabular}{|c|c|c|c|c|c|c|}
\hline \multirow{2}{*}{ Área } & \multicolumn{3}{|c|}{ Ítems por ámbitos de competencia } & \multicolumn{3}{|c|}{ Ítems por niveles de competencia } \\
\hline & Conocimiento & Habilidad & Actitud & Básico & Intermedi & Avanzado \\
\hline $\begin{array}{l}\text { A2. Comunicación y } \\
\text { colaboración }\end{array}$ & 8 & 10 & 6 & 7 & 8 & 3 \\
\hline
\end{tabular}

Una vez que se obtuvo el permiso de las autoridades de la Administración Educativa y del Comité ético de la Universidad de Salamanca, la aplicación de la prueba de evaluación se realizó por medio de un sitio web diseñado ad hoc para facilitar su cumplimentación por parte de los escolares. Todos los centros colaboraron de manera voluntaria y se encargaron de obtener los permisos de las familias, de los niños y de aplicar la prueba en el horario lectivo, siempre siguiendo las pautas y protocolos preparados por los investigadores. 


\subsection{Variables de estudio}

Se definieron las siguientes variables:

1) Variable dependiente: nivel (bajo, intermedio o alto) de competencia digital (conocimiento, habilidad y actitud) en el área de comunicación y colaboración, que presentan los estudiantes de Educación Obligatoria.

2) Variable independiente: nivel económico-cultural de la familia.

3) Variables mediadoras: cantidad de dispositivos tecnológicos y frecuencia de uso de los mismos.

4) Variables moderadoras: integración social y pertenencia a una familia estructurada.

Se pretende verificar la influencia del nivel económico-cultural de la familia (variable independiente) sobre el nivel de competencia digital en el área de comunicación y colaboración (variable dependiente), por medio de la disposición de dispositivos y frecuencia de uso de los mismos (variables mediadoras) y moderado por la Integración social y pertenecer a una familia estructurada (variables moderadoras). Todas ellas se recogen en la Figura 3.

Figura 3. Variables de estudio

VARIABLE DEPENDIENTE: nivel (bajo, intermedio o alto) de competencia digital (conocimiento, habilidad y actitud) en el área de comunicación y colaboración, que presentan los estudiantes de Educación Obligatoria.

VARIABLE INDEPENDIENTE: nivel económico-cultural de la familia.

VARIABLES MEDIADORAS: cantidad de dispositivos tecnológicos y

frecuencia de uso de los mismos.

VARIABLES MODERADORAS: integración social y pertenencia a una familia estructurada.

\subsection{Análisis de los datos}

El análisis de los datos se dirigió a conocer si existían diferencias significativas en los resultados de la evaluación de la competencia digital en el área de Comunicación y colaboración debido a la influencia de variables sociofamiliares. Para ello se realizó un análisis de regresión para comprobar las hipótesis planteadas.

Se utilizó la macro PROCESS v.3 para el programa SPSS v.25 (Hayes, 2018). Este software actúa como una ampliación de SPSS y permite realizar análisis de regresión mediante la creación de modelos teóricos de referencia. Esta herramienta hizo posible probar diferentes modelos de mediación basados en la técnica bootstrapping y sirvió, a su vez, para calcular el efecto de una variable independiente a partir del cálculo de los efectos indirectos condicionales.

Para el análisis de mediación moderada los efectos indirectos condicionales se calcularon utilizando 10000 muestras de bootstrap, generando intervalos de confianza del tipo bootstrap con corrección de sesgos. En concreto, se propusieron tres modelos: 
1) Modelo de mediación. Se pretende comprobar la influencia que ejerce el nivel económico y cultural de las familias en la adquisición de la competencia digital en el área de comunicación y colaboración, actuando como variables mediadoras la cantidad de dispositivos que tienen y la frecuencia de uso de los mismos.

2) Modelo de moderación. Se pretende verificar si el nivel económico-cultural de la familia influye en la adquisición de la competencia digital en el área de comunicación y colaboración, actuando como variables moderadoras la integración social del estudiante y la pertenencia a una familia estructurada.

3) Modelos de mediación moderada. Compuestos por los modelos A y el B. En el primero, se pretende confirmar una relación positiva entre el nivel económico y cultural de la familia y la adquisición de la competencia digital en el área de Comunicación y colaboración, con una variable de mediación (cantidad de dispositivos tecnológicos) y una moderadora (pertenencia a una familia estructurada). En el B se estudian las mismas variables que en el A, pero introduciendo la variable moderadora: actitud de los estudiantes hacia la tecnología.

\section{Resultados}

Teniendo en cuenta los datos descriptivos de las variables medidas, los estudiantes presentan un nivel medio de competencia digital en el área de Comunicación y colaboración $(\bar{x}=9.65)$, poseen muchos dispositivos digitales $(\bar{x}=5.70)$ con una frecuencia de uso media-alta $(\bar{x}=13.55)$, y pertenecen a familias estructuradas con un nivel económico-cultural medio-alto ( $\bar{x}=3.04$ ) (ver Tabla 3).

Tabla 3. Análisis descriptivo de las variables

\begin{tabular}{lccccc}
\hline & N & Mín & Máx & $\overline{\mathbf{x}}$ & DT \\
\hline NCDT & 609 & 2 & 17 & 9.65 & 2.87 \\
DD & 609 & 1 & 8 & 5.70 & 1.47 \\
IS & 609 & 1 & 4 & 1.95 & 0.22 \\
NECF & 609 & 1 & 4 & 3.04 & 0.72 \\
FUSOD & 609 & 2 & 21 & 13.55 & 2.15 \\
\hline Nota. NCDT: Nivel de & Competencia Digán
\end{tabular}

Nota. NCDT: Nivel de Competencia Digital Total; DD: Disposición de Dispositivos; IS: Integración Social; NECF: Nivel Económico y Cultural de la Familia; FUSOD: Frecuencia de Uso de Dispositivos.

De acuerdo con los modelos propuestos, enseguida se presentan los resultados obtenidos.

\subsection{Modelo de mediación}

Un modelo de mediación es un conjunto de dos o más variables causales encadenadas en secuencia $(\mathrm{X} / \mathrm{M} / \mathrm{Y})$. De tal manera que la variable mediadora $(\mathrm{M})$ debe estar localizada causalmente entre $\mathrm{X}$ e $\mathrm{Y}$, ser afectada por $\mathrm{X}$ y ésta, a su vez, debe afectar a Y.

La hipótesis 1 postula un modelo de mediación con la finalidad de entender la influencia que ejerce el nivel económico y cultural de las familias en el nivel de competencia digital adquirido por los estudiantes de Enseñanza Obligatoria, teniendo en cuenta la cantidad de dispositivos que tienen y la frecuencia de uso de los mismos, actuando estas últimas como variables mediadoras (M) del efecto de la similitud (ver Figura 4). 
Figura 4. Esquema Modelo 6 de mediación

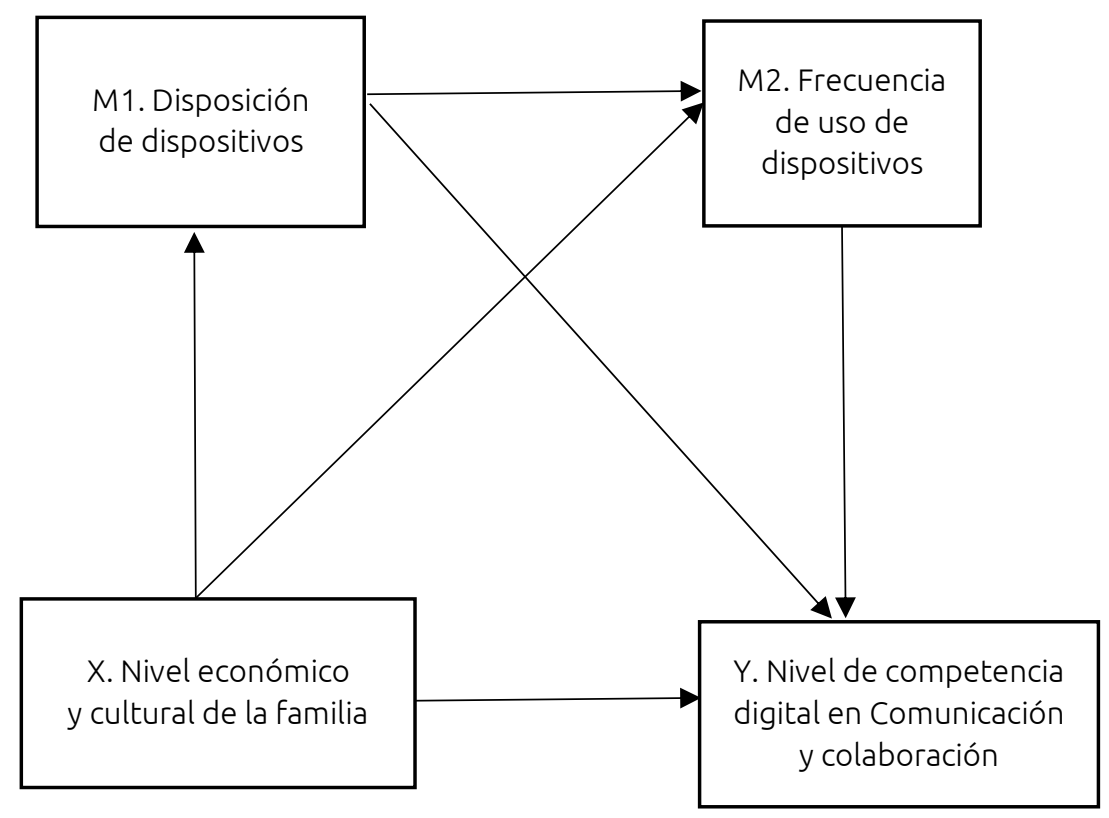

A la vista de los datos se puede decir que hay variables que afectan el nivel de competencia digital en el área de comunicación y colaboración. La variable independiente Nivel cultural y económico de la familia influye en la adquisición y desarrollo de esta competencia.

Además, el efecto moderador del nivel económico y cultural de la familia sobre la frecuencia de uso de dispositivos es tendencial o marginalmente significativo ( $B=0.2200 ; S E=0.1215, p=0.070)$ (Tabla 4).

Tabla 4. Análisis mediación. Relación Variable Independiente con Variable mediadora FUSOD

\begin{tabular}{lcccccc}
\hline \multicolumn{1}{c}{ FUSOD } & $\mathrm{B}$ & $\mathrm{SE}$ & $\mathrm{t}$ & $p$ & $\mathrm{LLCl}$ & $\mathrm{ULCl}$ \\
\hline constant & 13.0504 & .2910 & 44.8503 & .0000 & 12.4790 & 13.6219 \\
NECF $\rightarrow$ FUSOD & .2200 & .1215 & 1.8113 & .0706 & .0185 & .4586 \\
Standardized coefficients & $\mathrm{B}$ & & & & & \\
NECF & .0733 & & & & & \\
\hline
\end{tabular}

Nota. FUSOD: Frecuencia de uso de dispositivos; NECF: Nivel Económico y Cultural de la Familia.

Sin embargo, en la segunda variable moderadora objeto de estudio, el nivel económico y cultural de la familia afecta positivamente tanto a la disposición de dispositivos por parte de los estudiantes $(B=0.1616$; $S E=0.764, p=0.034)$ como a la frecuencia de uso de los mismos $(B=0.2761 ; S E=0.0255, p=0.00)($ Tabla 5). Por lo que se puede interpretar que tener un nivel cultural y económico familiar alto está relacionado con tener un mayor número de dispositivos tecnológicos propios y con mayor frecuencia de uso.

Tabla 5. Análisis mediación. Relación Variable Independiente con variables mediadoras FUSOD y DD

\begin{tabular}{lcccccc}
\hline Disposición de Dispositivos & B & SE & $t$ & $p$ & LLCl & ULCl \\
\hline constant & 1.5932 & .3790 & 4.2040 & .0000 & .8489 & 2.3374 \\
NECF $\rightarrow$ DD & .1616 & .0764 & 2.1154 & .0348 & .0116 & .3116 \\
DD $\rightarrow$ FUSOD & .2761 & .0255 & 10.8476 & .0000 & .2261 & .3261 \\
Standardized coefficients & $\mathrm{B}$ & & & & & \\
NECF & .0784 & & & & \\
FUSOD & .4020 & & & & \\
\hline Nota. DD: Disposición de dispositivos; FUSOD: Frecuencia de uso de dispositivos; NECF: Nivel Económico y cultural de la \\
familia.
\end{tabular}


Por su parte, el nivel de competencia digital está determinado significativamente por el nivel cultural y económico de la familia $(B=0.4231 ; S E=0.1609, p=0.008)$ y por la disposición de dispositivos $(B=0.2691$; $S E=0.0853, p=0.001)$. En cambio, no hay una relación significativa entre el nivel de competencia digital y la frecuencia de uso de dispositivos $(B=0.0358 ; S E=0.0584, p=0.539)$ (ver Tabla VI).

Tabla 6. Análisis mediación. Relación Variable Dependiente con Variable Independiente y variables mediadoras FUSOD y DD

\begin{tabular}{|c|c|c|c|c|c|c|}
\hline Nivel de Competencia Digital & B & SE & $\mathrm{t}$ & $p$ & $\mathrm{LLCl}$ & ULCI \\
\hline constant & 6.6658 & .8069 & 8.2611 & .0000 & 5.0812 & 8.2505 \\
\hline $\mathrm{NECF} \rightarrow \mathrm{NCD}$ & .4231 & 1609 & 2.6299 & .0088 & .1072 & .7391 \\
\hline FUSOD $\rightarrow$ NCD & .0358 & .0584 & .6136 & .5397 & -.0788 & .1505 \\
\hline $\mathrm{DD} \rightarrow \mathrm{NCD}$ & .2691 & .0853 & 3.1564 & .0017 & .1017 & .4365 \\
\hline Standardized coefficients & B & & & & & \\
\hline NECF & .1056 & & & & & \\
\hline FUSOD & .0268 & & & & & \\
\hline DD & .1384 & & & & & \\
\hline
\end{tabular}

Nota. NCD: Nivel de competencia digital; DD: Disposición de dispositivos; FUSOD: Frecuencia de uso de dispositivos; NECF:

Nivel Económico y cultural de la familia.

Los resultados muestran que se establece un efecto total en el modelo propuesto $(B=0.4909 ; S E=0.1615$; $p=0.002$, Cl 95\% [0.1737 - 0.8080]), lo que indica la influencia positiva de las dos variables mediadoras (disposición de dispositivos y frecuencia de uso de los mismos). También, que el nivel económico y cultural de la familia afecta significativamente al nivel de competencia digital en el área de comunicación y colaboración ( $B=0.4231$; $S E=0,1609 ; p=0.008, C / 95 \%$ [0.1072 - 0.7391]), estableciéndose un efecto directo entre la variable dependiente e independiente. Se puede afirmar, por tanto, que los estudiantes de Educación Obligatoria, cuyas familias tienen un mayor nivel económico y cultural, demuestran mejor competencia digital en el área de Comunicación y colaboración (Tabla 7).

Tabla 7. Efecto total del modelo, efecto directo entre las variables Dependiente e Independiente y efectos indirectos

\begin{tabular}{lccccc}
\hline Total effect of $X$ on $Y$ & $\mathrm{~B}$ & $\mathrm{SE}$ & $\mathrm{t}$ & $\mathrm{P}$ & $\mathrm{Boot} 95 \% \mathrm{Cl}$ \\
\hline NECF $\rightarrow \mathrm{DD} \rightarrow \mathrm{FUSOD} \rightarrow \mathrm{NCD}$ & .4909 & .1615 & 3.0398 & .0025 & {$[0.1737,0.8080]$} \\
Direct effect of $X$ on $\mathrm{Y}$ & $\mathrm{B}$ & $\mathrm{SE}$ & $\mathrm{t}$ & $\mathrm{p}$ & Boot $95 \% \mathrm{Cl}$ \\
NECF $\rightarrow$ NCD & .4231 & .1609 & 2.6299 & .0088 & {$[0.1072,0.7391]$} \\
\hline
\end{tabular}

Nota. NECF: Nivel Económico y cultural de la familia; DD: Disposición de dispositivos; FUSOD: Frecuencia de uso de dispositivos; NCD: Nivel de competencia digital.

Se producen tres efectos indirectos (bootstrapping) en la interrelación de variables: Efecto indirecto 1. a1b1 (ind1): $\mathrm{X} \rightarrow \mathrm{M} 1 \rightarrow \mathrm{Y}(B=0.0598, S E=0.0315, C / 95 \%$ [ 0.0094, 0.1311]; efecto indirecto 2. a2b2 (ind2): $\mathrm{X} \rightarrow$ $\mathrm{M} 2 \rightarrow \mathrm{Y}(B=0.0032, S E=0.0096, C / 95 \%[-0.0156,0.0252]$; efecto indirecto 3. a1d21b2 (ind3): $\mathrm{X} \rightarrow \mathrm{M} 1 \rightarrow$ $\mathrm{M} 2 \rightarrow \mathrm{Y}(B=0.0047, S E=0.0088, C / 95 \%[-0.0115,0.0242]$. Estos datos indican que se encuentra un efecto indirecto significativo cuando se relaciona el nivel económico y cultural de la familia con el nivel de competencia digital, mediado por la disposición de dispositivos digitales. Aquellos estudiantes procedentes de familias con mayor nivel económico y cultural disponen de más dispositivos propios y esto influye positivamente en un mejor nivel de competencia en Comunicación y colaboración. Sin embargo, cuando se incluye la segunda variable mediadora (frecuencia de uso de dispositivos tecnológicos), estos efectos no son significativos. De tal manera que la frecuencia de uso de dispositivos no actúa como una variable influyente en el nivel de competencia digital de los estudiantes.

Los contrastes que resultan de la comparación de los dos mediadores que se proponen en el modelo no son significativos en ningún caso (Tabla 8). 
Tabla 8. Contrastes de la comparación de los dos mediadores

\begin{tabular}{lccc}
\hline & B & SE & Boot 95\% Cl \\
\hline (C1) & -.0356 & .0332 & {$[-0.1085 ; 0.0226]$} \\
(C2) & -.0085 & .0196 & {$[-0.0556 ; 0.0247]$} \\
(C3) & .0271 & .0272 & {$[-.0227 ; 0.0879]$} \\
\hline
\end{tabular}

\subsection{Modelo de moderación}

Para comprobar la H2 se realiza un análisis de moderación y se utiliza el modelo 3 de moderación (Hayes, 2018) para establecer las relaciones entre las variables. Se percibe la influencia de dos variables moderadoras (integración social y estructura familiar) en las variables dependiente e independiente (ver Figura 5). Lo que se pretende comprobar es si el nivel económico-cultural de la familia influye en el nivel de competencia digital, moderado por la integración social del estudiante y por la pertenencia a una familia estructurada.

Figura 5. Esquema Modelo 3 de moderación

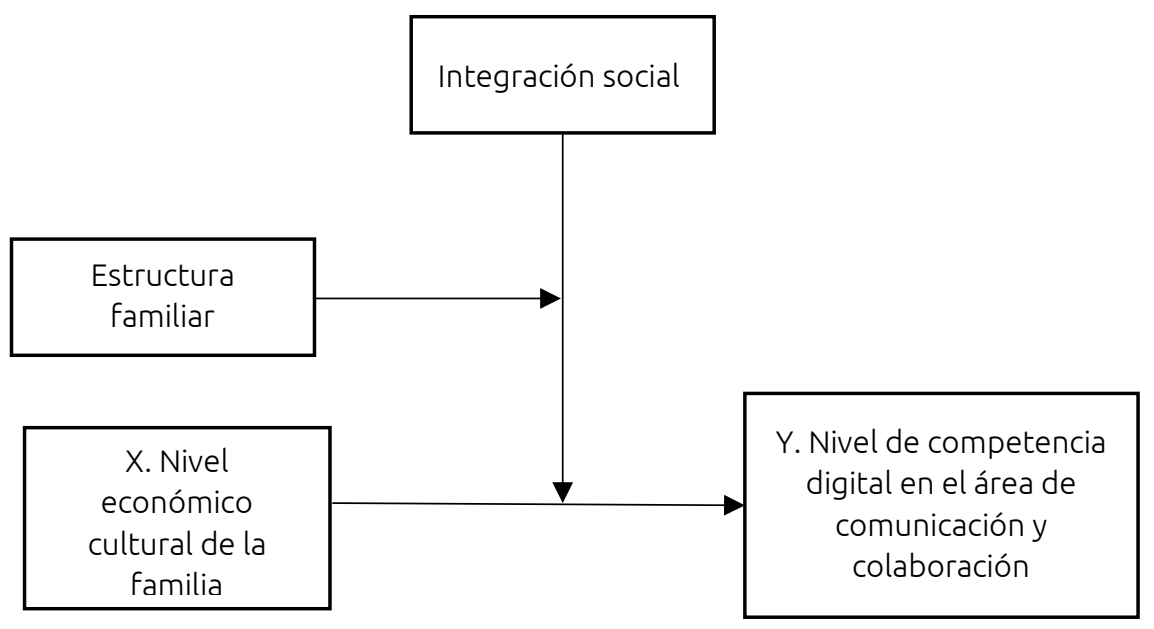

Los resultados obtenidos muestran que: a mayor nivel económico y cultural de las familias los estudiantes demuestran un mejor nivel de competencia digital, aunque con diferencias no significativas $(B=-0.8475, p$ $=0.630)$. Además, si el estudiante está mejor integrado en la sociedad $(B=-3.0793, P=0.500)$ y pertenece a una familia estructurada $(B=-0.7625, p=0.471)$, tendrá una mejor competencia, aunque las diferencias encontradas no son significativas (Tabla 9).

Tabla 9. Análisis moderación. Relación Variable Independiente, Variable Dependiente y Variable moderadora FE

\begin{tabular}{lcccccc}
\hline Model & \multicolumn{7}{c}{ LLCl } & ULCI \\
\hline NCD & coeff & SE & $t$ & $p$ & L & \\
\hline constant & 9.9045 & 3.7297 & 2.6556 & .0081 & 2.5796 & 17.2293 \\
NECF & -.8475 & 1.7589 & .4818 & .6301 & -4.3018 & 2.6068 \\
FE & -.7625 & 1.0590 & -.7200 & .4718 & -2.8424 & 1.3173 \\
IS & -3.0793 & 4.5643 & -.6746 & .5002 & -12.0433 & 5.8847 \\
\hline Nota. NCD: Nivel de Competencia Digital; NECF: Nivel Económico y Cultural de la Familia; \\
FE: Familia Estructurada, IS: Integración Social.
\end{tabular}

En la Figura 6 se pueden ver las diferencias encontradas en función de la variable moderadora relacionada con la variable de familias estructuradas. Los estudiantes de Educación Obligatoria que pertenecen a familias estructuradas consiguen un mejor resultado en la evaluación de su competencia digital en el área de comunicación y colaboración. 
Figura 6. Gráfico análisis moderación

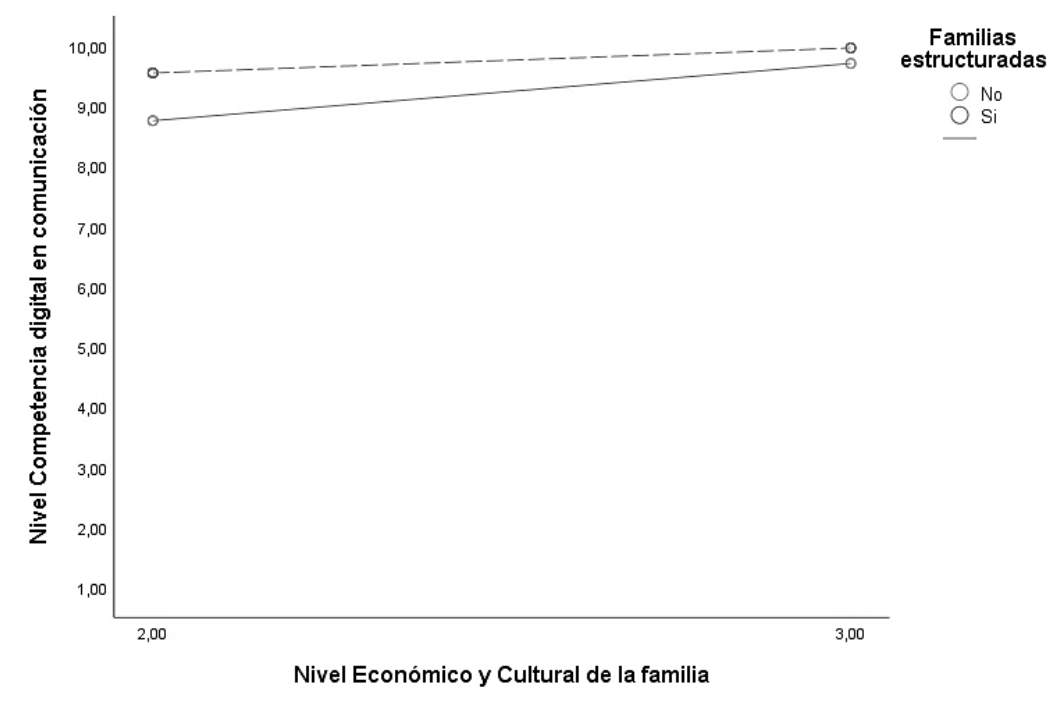

\subsection{Modelos de mediación moderada}

Se empleó de nuevo la macro PROCESS v.3 de SPSS para probar este modelo de mediación moderada propuesto, de acuerdo con la H3 de este estudio. En esta ocasión se utilizó el modelo 7 de mediación moderada (Hayes, 2018). El modelo A propuesto (ver Figura 7) plantea una relación positiva entre el nivel económico y cultural de la familia y el nivel de competencia digital en el área de Comunicación y colaboración; todo ello con la variable de mediación disposición de dispositivos y la variable moderadora pertenencia a una familia estructurada. A mayor nivel económico y cultural de la familia se dispone de más dispositivos y ello influye en tener un mejor nivel de competencia digital en esta área, influyendo también el hecho de pertenecer o no a familias estructuradas.

Figura 7. Modelo A: Esquema Modelo 7 de mediación moderada

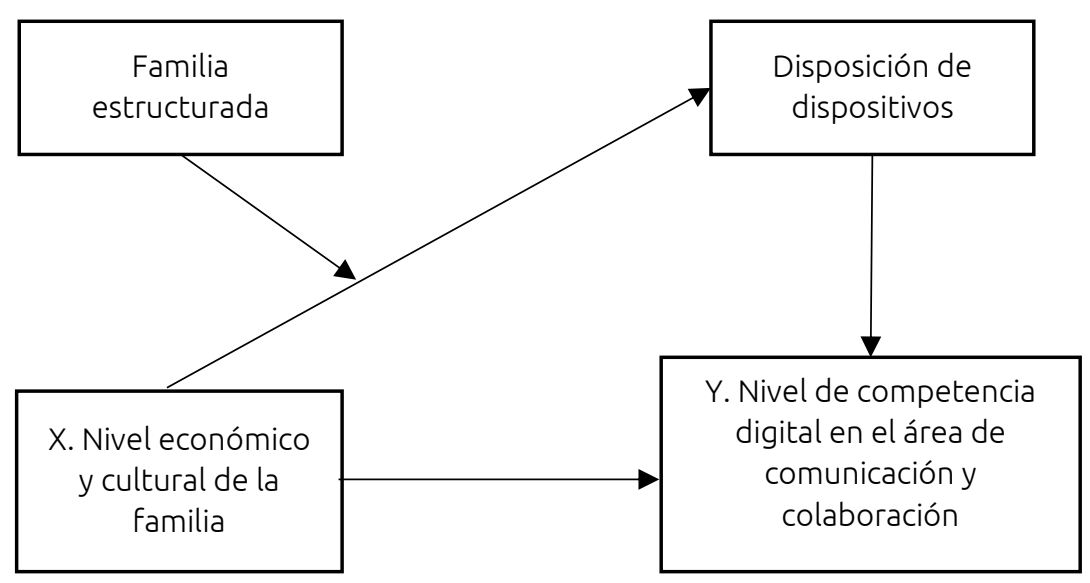

Existe una relación significativa entre las variables disposición de dispositivos y nivel económico y cultural de la familia $(B=0.61 ; S E=0.287 ; p=0.032)$, también entre disposición de dispositivos y pertenencia a una familia estructurada ( $B=1.578 ; S E=0.661 ; P=0.017$ ). En ambas relaciones de variables se encuentra una diferencia significativa (Tabla 10). 
Tabla 10. Análisis mediación moderada. Relación entre las variables (DD, NECF, FEST)

\begin{tabular}{lcccccc}
\hline Model & \multicolumn{7}{c}{ Loeff } & SE & $t$ & $p$ & LLCI & ULCI \\
\cline { 2 - 6 } & coency & .6275 & 6.0202 & .0000 & 2.5452 & 5.0097 \\
\hline constant & 3.7774 & .2879 & 2.1401 & .0327 & .0507 & 1.1815 \\
NECF $\rightarrow$ DD & .6161 & .6614 & 2.3868 & .0173 & .2797 & 2.8774 \\
FEST $\rightarrow$ DD & 1.5786 & .3007 & -1.5009 & .1339 & -1.0420 & .1393 \\
Int1=NECFxFEST & -.4514 & .300 &
\end{tabular}

A continuación se muestra una representación gráfica de las diferencias encontradas en este modelo de mediación moderada. Los resultados demuestran que los alumnos de familias estructuradas tienen mayor número de dispositivos a su alcance que aquellos que pertenecen a familias desestructuradas. Además, las familias que tienen mayor nivel económico y cultural son las que disponen de mayor número de dispositivos propios (Figura 8).

Figura 8. Gráfico análisis mediación moderada. Modelo A

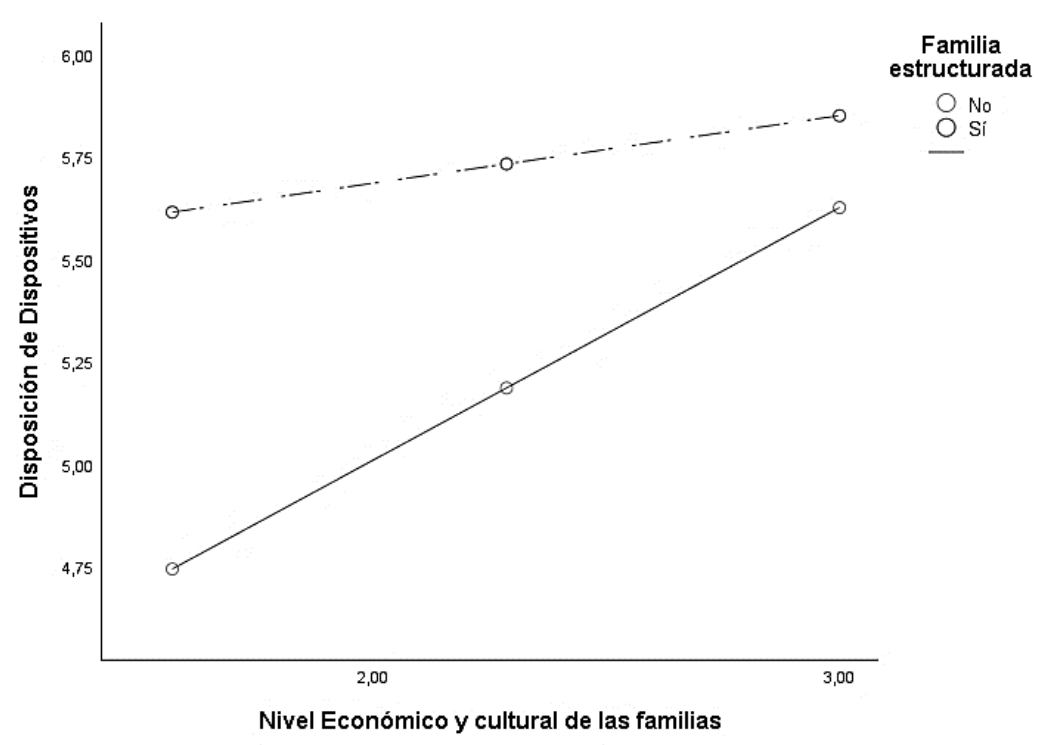

El nivel cultural y económico de la familia se asocia con mayor disposición de dispositivos en la unidad familiar y ésta, a su vez, con la pertenencia a una familia estructurada pero ningún efecto indirecto condicional resultó ser estadísticamente significativo y, por lo tanto, el índice de mediación moderada tampoco fue estadísticamente significativo (IMM =-0.131), $S E=0.120, C / 95 \%[-0.387,0.083])$. Aunque las variables están relacionadas, las diferencias encontradas no son significativas.

En el modelo B (Figura 9) la variable moderadora es la actitud de los estudiantes hacia la competencia digital en el área de Comunicación y colaboración. Pretende comparar la relación entre el nivel económico y cultural de la familia y el nivel de competencia digital, mediada por la disposición de dispositivos y como variable moderadora la actitud hacia la tecnología. 
Figura 9. Modelo B: Esquema Modelo 7 de mediación moderada

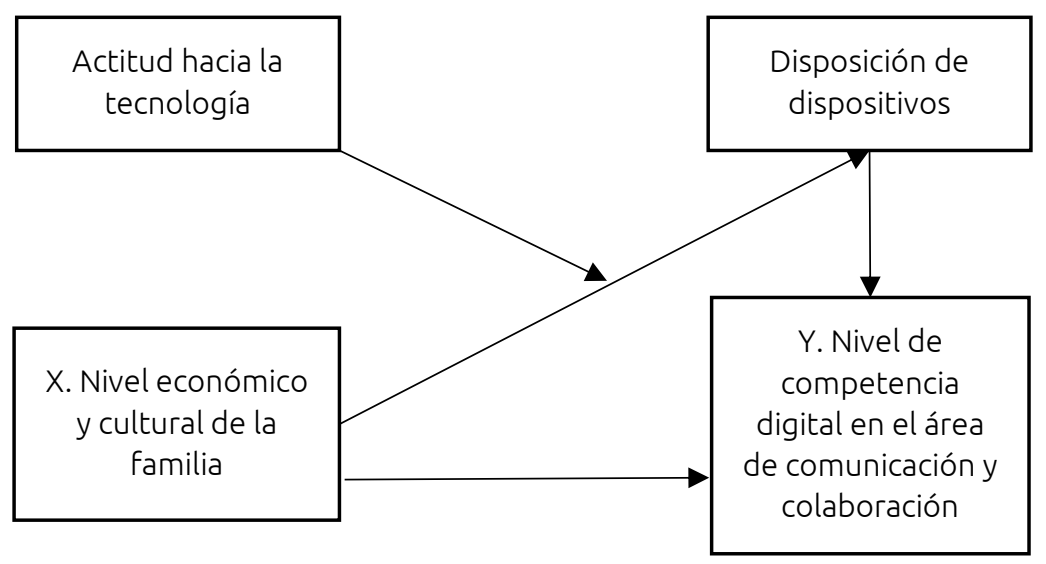

Como se puede ver en la Tabla 11, existe relación entre el nivel económico y cultural de la familia y la disposición de dispositivos. También entre disposición de dispositivos y actitud hacia la tecnología. Sin embargo, estas diferencias no son significativas en ningún caso a nivel 0.05.

Tampoco se encuentran diferencias significativas cuando se relaciona el nivel económico y cultural de la familia con la actitud hacia la tecnología.

Tabla 11. Análisis de mediación moderada. Relación entre variables (DD, NECF, ACTITUD)

\begin{tabular}{lcccccc}
\hline Model & \multicolumn{7}{c}{} \\
\cline { 2 - 6 } & coeff & se & t & P & LLCl & ULCl \\
\hline constant & 4.9010 & .9081 & 5.3968 & .0000 & 3.1175 & 6.6845 \\
NECF $\rightarrow$ DD & -.1097 & .3887 & -.2822 & .7779 & -.8730 & .6536 \\
ACTITUD $\rightarrow$ DD & .0124 & .0352 & .3510 & .7257 & -.0569 & .0816 \\
Int_1 NECFxACTITUD & .0124 & .0150 & .8265 & .4088 & -.0170 & .0418 \\
\hline
\end{tabular}

Nota. DD: Disposición de dispositivos, NECF: Nivel Económico y cultural de la familia.

La Figura 10 muestra que los que tienen mejor actitud disponen de mayor número de dispositivos.

Figura 10. Gráfico análisis mediación moderada. Modelo B

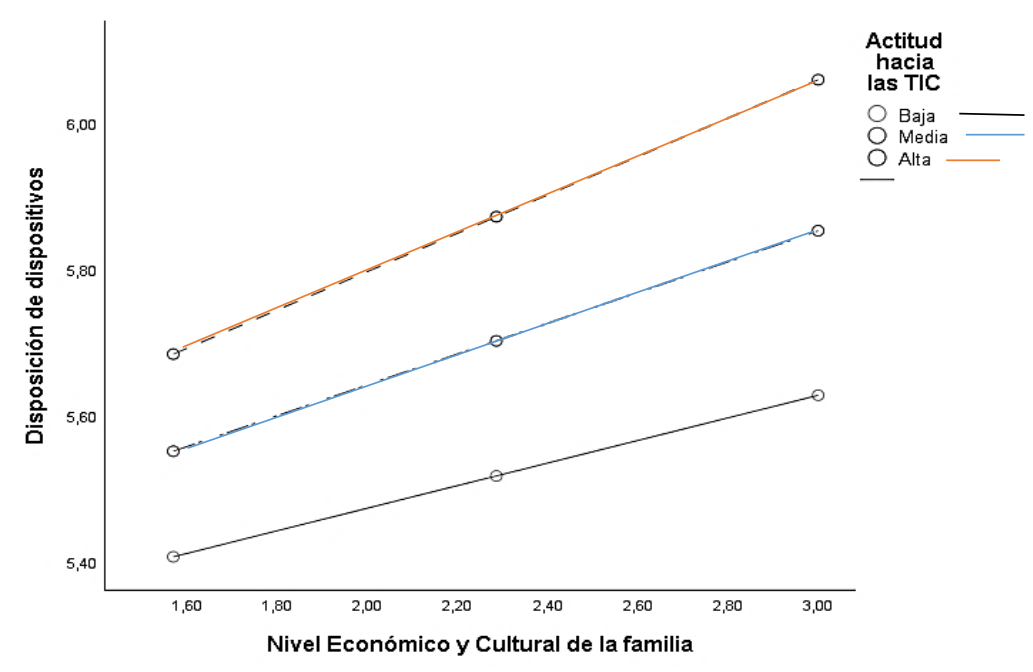


Existe un efecto condicional indirecto entre los que tienen una actitud media o alta, a diferencia de los que la tienen baja, en los que no se encuentran diferencias significativas (ver Tabla 12) de relación con estas variables. Por tanto, se podría interpretar que los que tienen una actitud más positiva son los que presentan un mejor nivel de competencia digital en el área de comunicación y colaboración. El Índice de Mediación Moderada no es significativo (IMM = -0.0036), SE=0.0067, Cl 95\% [-0.0051, 0.0215]).

Tabla 12. Efecto condicional indirecto de $X$ en $Y$

\begin{tabular}{lcccc}
\hline NECF $\rightarrow$ DD $\rightarrow$ NCD & & & & \\
\hline ACTITUD & Effect & BootSE & BootLLCl & BootULCl \\
\hline BAJA & .0447 & .0410 & .0489 & .1177 \\
MEDIA & .0610 & .0313 & .0085 & .1293 \\
ALTA & .0760 & .0446 & .0101 & .1824 \\
\hline
\end{tabular}

Nota. NECF: Nivel Económico y Cultural de la Familia; DD: Disposición de Dispositivos; NCD: Nivel de Competencia Digital.

\section{Discusión y conclusiones}

En esta investigación se han identificado modelos sobre la influencia de variables sociofamiliares en la evaluación de la competencia digital en el área de Comunicación y colaboración, de estudiantes españoles de Educación Obligatoria (12-16 años), teniendo en cuenta sus conocimientos, capacidades y actitudes. Se ha demostrado que estas variables influyen en la adquisición y desarrollo de la competencia digital, al igual que se recoge en Centeno y Cubo (2013), y Hatlevik y Christophersen (2013).

Si tenemos en cuenta la primera hipótesis planteada, se puede confirmar que el nivel cultural y económico de la familia influye en la adquisición y desarrollo de la competencia digital en el área de Comunicación y colaboración, lo que corrobora lo ya constatado en otras investigaciones, en las que se manifiesta que esta variable es un predictor muy importante de la competencia digital de los jóvenes (Aesaert y van Braak, 2014; Ames, 2016; Claro et al., 2015; Segura et al., 2007; van Deursen y van Diepen, 2013). Esta capacidad está relacionada significativamente con el nivel cultural y económico de la familia y con la disposición de dispositivos tecnológicos. Así, los estudiantes que pertenecen a familias cuyo nivel cultural y económico es alto y tienen más dispositivos tecnológicos demuestran una mejor competencia. Este hecho contrasta con los resultados encontrados en Colás et al. (2017, p. 18) en la que se llega a la conclusión de que "no es tan importante la exposición a diferentes medios o dispositivos para desarrollar niveles deseables de competencia digital, sino un acercamiento temprano al ordenador y sus posibilidades en Internet como vehículo para la adquisición de habilidades digitales globales".

Por su parte, Pérez-Escoda et al. (2016, p. 77) manifiestan que "la mera exposición, uso y convivencia con los medios y la tecnología, no supone el desarrollo de la competencia digital". También Casillas-Martín y Cabezas-González (2019) evidencian que los estudiantes que poseen más dispositivos TIC no son más competentes para manejarlos ni tienen mayores conocimientos sobre tecnología. Si tenemos en cuenta la frecuencia en el uso de los dispositivos, son muchos los trabajos que evidencian su efecto positivo en la competencia digital (Almerich et al., 2018; Almerich et al., 2020; OECD, 2011; Zhong 2011) frente a otros que muestran su ausencia (van Deursen y van Diepen, 2013; Vekiri y Chronaki, 2008). En el caso que nos ocupa las diferencias encontradas no son significativas, por lo que no se puede confirmar esta influencia.

En cuanto a la segunda hipótesis, a diferencia de otras investigaciones, como la de Kuhlmeier y Hemker (2007), no se puede confirmar que aquellos alumnos que socialmente están integrados y que pertenecen a familias estructuradas con un nivel cultural y económico alto tengan una competencia digital mejor, pues las diferencias encontradas no son significativas.

En la tercera hipótesis se confirma la relación significativa entre la disposición de dispositivos y el nivel económico y cultural de la familia; también entre la disposición de dispositivos y la pertenencia a una familia estructurada. Se puede concluir, de acuerdo con Kuhlmeier y Hemker (2007), que las familias estructuradas y con un mayor nivel económico y cultural son las que tienen más dispositivos y ello influye en que la competencia digital de los alumnos sea mejor. Por otro lado, existe una relación positiva, aunque no significativa, entre el nivel económico y cultural de la familia, la disposición de dispositivos y la actitud hacia 
la tecnología, en el sentido de que a mayor nivel económico y cultural, más disposición de dispositivos y mejor actitud hacia la tecnología. Tampoco se encuentran diferencias significativas cuando se relaciona el nivel económico y cultural de la familia con la actitud hacia la tecnología. Por último, se puede concluir que los estudiantes que tienen una actitud más positiva demuestran una mejor competencia digital.

En la actual sociedad digital es clave adquirir y desarrollar competencias digitales para poder ejercer una participación social plena y conseguir una mayor movilidad social (Sánchez-Antolín et al., 2018). Por ello, este trabajo contribuye al conocimiento del impacto que pueden tener determinadas variables sociofamiliares en la adquisición y desarrollo de la competencia digital, para favorecer la puesta en práctica de políticas de integración de las TIC en los centros educativos.

\section{Referencias}

Aesaert, K. y van Braak, J. (2014). Exploring factors related to primary school pupils' ICT self-efficacy: A multilevel approach. Computers in Human Behavior, 41, 327-341. https://doi.org/10.1016/i.chb.2014.10.006

Almerich, G., Díaz-García, l., Cebrián-Cifuentes, S. y Suárez-Rodríguez, J. M. (2018). Estructura dimensional de las competencias del siglo XXI en alumnado universitario de educación. RELIEVE, 24(1), 1-21.

https://doi.org/10.7203/relieve.24.1.12548

Almerich, G., Suárez-Rodríguez, J., Díaz-García, I. y Orellana, N. (2020). Estructura de las competencias del siglo XXI en alumnado del ámbito educativo. Factores personales influyentes. Educación XX1, 23(1), 45-74. https://doi.org/10.5944/educXX1.23853

Álvarez-Quiroz, G. B. y Vélez, C. P. (2014). ¿Qué se ha hecho sobre las TIC en la educación superior y sobre la relación jóvenes y TIC? Revisión a investigaciones realizadas en América, Europa y Asia. Revista d'Innovació i Recerca en Educació, 7(2), 28-52. https://doi.org/10.1344/reire2014.7.2723

Ames, P. (2016). Los niños y sus relaciones con las tecnologías de información y comunicación: un estudio en escuelas peruanas. Revista Eletrónica de Divulgación Científica de la Infancia y la Juventud, (11), 11-21. http://desidades.ufri.br/wp-content/uploads/v11n1ES.pdf

Ávila, H. L. (2006). Introducción a la metodología de la investigación. Eumed.net.

Cabero, J. (2015). La tecnología como eje de cohesión y participación en la ciudad y en la ciudadanía. En A. Monclús y C. Sabán (Coords.), Ciudad y educación: antecedentes y nuevas perspectivas (pp. 155-170). Síntesis.

Cabero, J. y Ruiz-Palmero, J. (2018). Las tecnologías de la información y la comunicación para la inclusión: reformulando la brecha digital. International Journal of Educational Research and Innovation, 9, 16-30. https://www.upo.es/revistas/index.php/IJERI/article/view/2665

Calvani, A., Cartelli, A., Fini, A. y Ranieri, M. (2008). Models and instruments for assessing digital competence at school. Journal of e-Learning and Knowledge Society, 4(3), 183-193.

Carretero, S., Vuorikari, R. y Punie, Y. (2017). DigComp 2.1. The digital competence framework for citizens. Publications Office of the European Union. https://doi.org/10.2760/38842

Casal, J. y Mateu, E. (2003). Tipos de muestreo. Rev. Epidem. Med. Prev, 1(1), 3-7.

Casillas-Martín, S. y Cabezas González, M. (2019). ¿Están preparados los maestros de Infantil para educar en la Sociedad Red? Opción, 35(89-2), 1317-1348.

https://produccioncientificaluz.org/index.php/opcion/article/view/27528 
Centeno, G. y Cubo, S. (2013). Evaluación de la competencia digital y las actitudes hacia las TIC del alumnado universitario. Revista de Investigación Educativa, 31(2), 517-536. https://doi.org/10.6018/rie.31.2.169271

Claro, M., Cabello, T., San Martín, E. y Nussbaum, M. (2015). Comparing marginal effects of Chilean students' economic, social and cultural status on digital versus reading and mathematics performance. Computers and Education, 82, 1-10. https://doi.org/10.1016/i.compedu.2014.10.018

Colás, P., Conde, J. y Reyes, S. (2017). Digital competences of non-university students. Revista Latinoamericana de Tecnología Educativa, 16(1), 7-20. https://idus.us.es/handle/11441/61516

Erstad, O. (2010). Educating the digital generation. Nordic Journal of Digital Literacy, 5(1), 56-70. https://www.idunn.no/dk/2010/01/art05

Erstad, O. y Voogt, J. (2018). The twenty-first century curriculum: issues and challenges. En J. Voogt, G. Knezek, R. Christensen, y K.W. Lai (Eds.), Second handbook of information technology in primary and secondary education (pp. 19-36). Springer.

Fernández, M. y Manzano, D. (2018). Análisis de las diferencias en la competencia digital de los alumnos españoles. Papers, 103(2), 175-198. https://core.ac.uk/download/201379718.pdf

García, A., Hernández, A., Mena, J. J., Iglesias, A., Casillas-Martín, S., Cabezas-González, M., González, L. M., Martín del Pozo, M. y Basilotta, V. (2019). Modelo de indicadores para evaluar la competencia digital de los estudiantes tomando como referencia el modelo DICCOMP (INCODIES).

https://gredos.usal.es/handle/10366/139409

Hargittai, E. (2010). Digital na(t)ives? Variation in Internet skills and uses among members of the 'Net Generation'. Sociological Inquiry, 80(1), 92-113. https://doi.org/10.1111/j.1475-682X.2009.00317.x

Hatlevik, O. E. y Christophersen, K. A. (2013). Digital competence at the beginning of upper secondary school: Identifying factors explaining digital inclusion. Computers \& Education, 63, 240-247.

https://doi.org/10.1016/i.compedu.2012.11.015

Hatlevik, O.E., Gudmundsdottir, G. y Loi, M. (2015). Digital diversity among upper secondary students: A multilevel analysis of the relationship between cultural capital, self-efficacy, strategic use of information and digital competence. Computers \& Education, (81), 345-353.

https://doi.org/10.1016/i.compedu.2014.10.019

Hatlevik, O.E., Ottestad, G. y Throndsent, I. (2014). Predictors of digital competence in 7th grade: a multilevel analysis. Journal of Computer Assisted Learning, 31, 220-231. https://doi.org/10.1111/ical.12065

Hayes, A. F. (2018). Introduction to mediation, moderation, and conditional process analysis second edition: A regression-based approach. The Guilford Press.

Kim, H. J., Hong, A. J. y Song, H. D. (2018). The relationships of family, perceived digital competence and attitude, and learning agility in sustainable student engagement in higher education. Sustainability, 10(12), 1-16. https://doi.org/10.3390/su10124635

Kuhlmeier, H. y Hemker, B. (2007). The impact of computer use at home on students internet skills. Computers \& Education, 49(2), 460-480. https://doi.org/10.1016/i.compedu.2005.10.004

Li, Y. y Ranieri, M. (2010). Are 'digital natives' really digitally competent? A study on Chinese teenagers. British Journal of Educational Technology, 41(6), 1029-1042. https://bera-journals.onlinelibrary.wiley.com Ldoi/10.1111/j.1467-8535.2009.01053.x 
Lorenz, R., Endberg, M. y Bos, W. (2019). Predictors of fostering students' computer and information literacy -analysis based on a representative sample of secondary school teachers in Germany. Education and Information Technologies, 24(1), 911-928. https://doi.org/10.1007/s10639-018-9809-0

McMillan, J. H. y Schumacher, S. (2005). Investigación educativa (5a. ed.). Pearson.

OECD. (2011). PISA 2009 Results: Students on line. Digital technologies and performance. Autor.

Pagani, L., Argentin, G., Gui, M. y Stanca, L. (2016). The impact of digital skills on educational outcomes: Evidence from performance tests. Educational Studies, 42(2), 137-162.

https://doi.org/10.1080/03055698.2016.1148588

Pérez-Escoda, A., Castro-Zubizarreta, A. y Fandos-Igado, M. (2016). Digital skills in the z generation: Key questions for a curricular introduction in primary school. Comunicar, 24(49), 71-79.

http://dx.doi.org/10.3916/C49-2016-07

Punter, R. A., Meelissen, M. R. y Glas, C. A. (2017). Gender differences in computer and information literacy: An exploration of the performances of girls and boys in ICILS 2013. European Educational Research Journal, 16(6), 762-780. https://doi.org/10.1177/1474904116672468

Rolf, E., Knutsson, O. y Ramberg, R. (2019). An analysis of digital competence as expressed in design patterns for technology use in teaching. British Journal of Educational Technology, 50(6), 1-15.

https://doi.org/10.1111/bjet.12739

Sánchez-Antolín, P., Andrés, C. y Paredes, J. (2018). El papel de la familia en el desarrollo de la competencia digital. Análisis de cuatro casos. Digital Education Review, (34), 44-58.

https://revistes.ub.edu/index.php/der/article/view/20750/pdf

Sefton, J., Nixon, H. y Erstad, O. (2009). Reviewing approaches and perspectives on "digital literacy". Pedagogies: An International Journal, 4(2), 107-125. http://dx.doi.org/10.1080/15544800902741556

Segura, M., Candioti, C. y Medina, J. (2007). Las TIC en la Educación: panorama internacional y situación Española. https://www.oei.es/historico/noticias/spip.php?article1383

Shala, A. y Grajcevci, A. (2018). Digital competencies among student populations in Kosovo: the impact of inclusion, socioeconomic status, ethnicity and type of residence. Education and Information Technologies, 23(3), 1203-1218. https://doi.org/10.1007/s10639-017-9657-3

Siddiq, F., Hatlevik, O.E., Olsen, R.V., Throndsen, I. y Scherer, R. (2016). Taking a future perspective by learning from the past. A systematic review of assessment instruments that aim to measure primary and secondary school students' ICT literacy. Educational Research Review, (19), 58-84.

https://doi.org/10.1016/i.edurev.2016.05.002

Torres, C. (2017). Sociedad de la Información y brecha digital en España. Panorama Social, (25), 17-33. https://www.funcas.es/wp-content/uploads/Migracion/Articulos/FUNCAS PS/025art03.pdf

UNESCO (2008). Estándares de competencia en TIC para docentes. Una propuesta en el contexto chileno. Centro de Educación y Tecnología del Ministerio de Educación de Chile.

https://unesdoc.unesco.org/ark:/48223/pf0000163149

Unión Europea (2018). Recomendación C 189, de 22 de mayo de 2018 relativa a las competencias clave para el aprendizaje permanente. Diario Oficial de la Unión Europea, 4 de junio de 2018. https://eurlex.europa.eu/legal-content/es/TXT/?uri=CELEX\%3A32018H0604\%2801\%29 
van Deursen, A. y van Diepen, S. (2013). Information and strategic internet skills of secondary students: A Performance Test. Computers \& Education, (63), 218-226. https://doi.org/10.1016/j.compedu.2012.12.007

Varela, J. (2015). La brecha digital en España. Estudio sobre la desigualdad postergada. Comisión Ejecutiva Confederal de UGT.

Vekiri, I. y Chronak i, A. (2008). Gender issues in technology use: perceived social support, computer selfefficacy and value beliefs, and computer use beyond school. Computers \& Education, 51(3), 1392-1404.

https://doi.org/10.1016/j.compedu.2008.01.003

Zakrajšek, S. (2018). Possible education models for acquiring digital competence of students. Didactica Slovenica, 33(3-4), 94-106. http://www.pedagoska-obzorja.si/revija/Vsebine/vs18-3.html

Zhong, Z. (2011). From access to usage: The divide of self-reported digital skills among adolescents. Computers \& Education, 56(3), 736-746. https://doi.org/10.1016/j.compedu.2010.10.016 